PROCEEDINGS OF THE

AMERICAN MATHEMATICAL SOCIETY

Volume 140, Number 10, October 2012, Pages 3623-3628

S 0002-9939(2012)11179-7

Article electronically published on February 20, 2012

\title{
MARTIN-LÖF RANDOM POINTS SATISFY BIRKHOFF'S ERGODIC THEOREM FOR EFFECTIVELY CLOSED SETS
}

\author{
JOHANNA N. Y. FRANKLIN, NOAM GREENBERG, JOSEPH S. MILLER, \\ AND KENG MENG NG
}

(Communicated by Julia Knight)

\begin{abstract}
We show that if a point in a computable probability space $X$ satisfies the ergodic recurrence property for a computable measure-preserving $T: X \rightarrow X$ with respect to effectively closed sets, then it also satisfies Birkhoff's ergodic theorem for $T$ with respect to effectively closed sets. As a corollary, every Martin-Löf random sequence in the Cantor space satisfies Birkhoff's ergodic theorem for the shift operator with respect to $\Pi_{1}^{0}$ classes. This answers a question of Hoyrup and Rojas.
\end{abstract}

Several theorems in ergodic theory state that almost all points in a probability space behave in a regular fashion with respect to an ergodic transformation of the space. For example, if $T: X \rightarrow X$ is ergodic 1 then almost all points in $X$ recur in a set of positive measure:

Theorem 1 (See [5]). Let $(X, \mu)$ be a probability space, and let $T: X \rightarrow X$ be ergodic. For all $E \subseteq X$ of positive measure, for almost all $x \in X, T^{n}(x) \in E$ for infinitely many $n$.

Recent investigations in the area of algorithmic randomness relate the hierarchy of notions of randomness to the satisfaction of computable instances of ergodic theorems. This has been inspired by Kučera's classic result characterising MartinLöf randomness in the Cantor space. We reformulate Kučera's result using the general terminology of [4.

Definition 2. Let $(X, \mu)$ be a probability space, and let $T: X \rightarrow X$ be a function. Let $\mathcal{C}$ be a collection of measurable subsets of $X$. A point $x \in X$ is a Poincaré point for $T$ with respect to $\mathcal{C}$ if for all $E \in \mathcal{C}$ of positive measure for infinitely many $n, T^{n}(x) \in E$.

The Cantor space $2^{\omega}$ is equipped with the fair-coin product measure $\lambda$. The shift operator $\sigma$ on the Cantor space is the function $\sigma\left(a_{0} a_{1} a_{2} \ldots\right)=a_{1} a_{2} \ldots$ The shift operator is ergodic on $\left(2^{\omega}, \lambda\right)$.

Received by the editors July 20, 2010 and, in revised form, April 5, 2011 and April 8, 2011.

2010 Mathematics Subject Classification. Primary 03D22; Secondary 28D05, 37A30.

The second author was partially supported by the Marsden Grant of New Zealand.

The third author was supported by the National Science Foundation under grants DMS0945187 and DMS-0946325, the latter being part of a Focused Research Group in Algorithmic Randomness.

${ }^{1}$ Recall that if $(X, \mu)$ is a probability space, then a measurable map $T: X \rightarrow X$ is measure preserving if for all measurable $A \subseteq X, \mu\left(T^{-1} A\right)=\mu(A)$. We say that a measurable set $A \subseteq X$ is invariant under a map $T: X \rightarrow X$ if $T^{-1} A=A$ (up to a null set). A measure-preserving map $T: X \rightarrow X$ is ergodic if every $T$-invariant measurable subset of $X$ is either null or conull. 
Theorem 3 (Kučera [7). A sequence $R \in 2^{\omega}$ is Martin-Löf random if and only if it is a Poincaré point for the shift operator with respect to the collection of effectively closed (i.e., $\Pi_{1}^{0}$ ) subsets of $2^{\omega}$.

Building on work of Bienvenu, Day, Mezhirov and Shen 2, Bienvenu, Hoyrup and Shen generalised Kučera's result to arbitrary computable ergodic transformations of computable probability spaces.

Theorem 4 (Bienvenu, Hoyrup and Shen [3]). Let $(X, \mu)$ be a computable probability space, and let $T: X \rightarrow X$ be a computable ergodic transformation. A point $x \in X$ is Martin-Löf random if and only if it is a Poincaré point for $T$ with respect to the collection of effectively closed subsets of $X 2$

One of the most fundamental regularity theorems is due to Birkhoff (see [5]).

Birkhoff's Ergodic Theorem. Let $(X, \mu)$ be a probability space, and let $T: X \rightarrow$ $X$ be ergodic. Let $f \in L^{1}(X)$. Then for almost all $x \in X$,

$$
\lim _{n \rightarrow \infty} \frac{1}{n} \sum_{i<n} f\left(T^{i}(x)\right)=\int f d \mu .
$$

Of particular interest is the case when $f$ is the characteristic function of a measurable subset of a space. Let $(X, \mu)$ be a probability space, and let $T: X \rightarrow X$ be a measurable function. For any $f \in L^{1}(X)$ and $n<\omega$ we let

$$
f^{(n)}(x)=\frac{1}{n} \sum_{i<n} f\left(T^{i}(x)\right)
$$

If $A \subseteq X$ is measurable, we let $1_{A}$ denote $A$ 's characteristic function, so for all $n<\omega$ and $x \in X$, we can define

$$
1_{A}^{(n)}(x)=\frac{\#\left\{i<n: T^{i}(x) \in A\right\}}{n} .
$$

Birkhoff's ergodic theorem implies that if $T$ is ergodic, then for almost all $x \in X$,

$$
\lim _{n \rightarrow \infty} 1_{A}^{(n)}(x)=\mu(A) .
$$

We can therefore make an analogue of Definition 2

Definition 5. Let $(X, \mu)$ be a probability space, and let $T: X \rightarrow X$ be a function. Let $\mathcal{C}$ be a collection of measurable subsets of $X$. A point $x \in X$ is a Birkhoff point for $T$ with respect to $\mathcal{C}$ if for all $E \in \mathcal{C}$,

$$
\lim _{n \rightarrow \infty} 1_{E}^{(n)}(x)=\mu(E) .
$$

\footnotetext{
${ }^{2}$ Here we use the notion of a computable probability space of Gács, Hoyrup and Rojas 4], although all reasonable definitions of this concept are equivalent. For completeness, we recall the definition here. A computable metric space is a complete metric space $(X, d)$ that is equipped with an enumeration $\left\langle s_{i}\right\rangle$ of a countable dense subset of $X$ such that $(i, j) \mapsto d\left(s_{i}, s_{j}\right)$ is a computable function. This enumeration gives rise to an enumeration $\left\langle B_{i}\right\rangle$ of basic open balls of $X$ that form a basis for its topology. A c.e. (computably enumerable) open subset of $X$ is a set of the form $\bigcup_{i \in S} B_{i}$ where $S \subseteq \omega$ is c.e. A function $f: X \rightarrow Y$ between two computable metric spaces is computable if, uniformly, the inverse of a c.e. open set is c.e. open. An effectively closed set is the complement of a c.e. open set. A Borel probability measure $\mu$ on $X$ is computable if, uniformly, the measure of a finite union of basic open balls is a left-c.e. real, equivalently, if it is a computable point in the space of Borel probability measures on $X$ equipped with the topology of weak convergence. A computable probability space is a computable metric space equipped with a computable measure.
} 
Gács, Hoyrup and Rojas [4] characterised the Schnorr random points as the Birkhoff points for computable ergodic transformations with respect to effectively closed sets whose measure is computable. They asked [8] what happens if we omit the requirement that the measure of the sets be computable.

Theorem 6. Let $X$ be a computable probability space, and let $T: X \rightarrow X$ be a computable ergodic map. Then a point $x \in X$ is Martin-Löf random if and only if it is a Birkhoff point for $T$ with respect to the collection of effectively closed subsets of $X$.

Of course if $x \in X$ is a Birkhoff point for $T$ with respect to $\mathcal{C}$, then it is a Poincaré point for $T$ with respect to $\mathcal{C}$. Our main result is an instance of the converse.

Theorem 7. Let $(X, \mu)$ be a computable probability space, and let $T: X \rightarrow X$ be a computable, measure-preserving transformation. Let $x \in X$ be a Poincaré point for $T$ with respect to the collection of effectively closed subsets of $X$. Then $x$ is also a Birkhoff point for $T$ with respect to the collection of effectively closed subsets of $X$.

Theorem 6 follows immediately from the combination of Theorems 7 and 4 together with the fact that any Birkhoff point is a Poincaré point. We should note that as the present paper was in preparation, Hoyrup independently announced a proof of Theorem 6. see [1.

We set about to prove Theorem 7. To prove a limit exists and has the required value, we discuss the partial limits, the inferior and superior, separately.

Proposition 8. Let $(X, \mu)$ be a computable probability space, and let $T: X \rightarrow X$ be a computable, measure-preserving transformation. Let $x \in X$ be a Poincaré point for $T$ with respect to the collection of effectively closed subsets of $X$. Then for any effectively closed subset $P$ of $X$,

$$
\liminf _{n} 1_{P}^{(n)}(x) \geqslant \mu(P) .
$$

We first prove Proposition 8 and then show that it implies the full Theorem 7 We need the concept of right-c.e. functions on a computable metric space. These are the effectively upper semi-continuous functions.

Definition 9. Let $X$ be a computable metric space. A function $f: X \rightarrow \mathbb{R}$ is right-c.e. if uniformly in $q \in \mathbb{Q}, f^{-1}(-\infty, q)$ is c.e. open in $X$.

Thus every computable function $f: X \rightarrow \mathbb{R}$ is right-c.e., but not every right-c.e. function is computable:

Example 10. Let $P \subseteq X$ be effectively closed. Then $1_{P}$ is right-c.e.: for $q \leqslant 0$, $1_{P}^{-1}(-\infty, q)=\emptyset$; for $q \in(0,1], 1_{P}^{-1}(-\infty, q)=X \backslash P$; and for $q>1,1_{P}^{-1}(-\infty, q)=X$. If $P$ is not c.e. open, then $1_{P}$ is not computable, since $1_{P}^{-1}(-1 / 2, \infty)=P$. Indeed, if $P$ is not clopen, then $1_{P}$ is not continuous, whereas every computable function is continuous.

Lemma 11. Let $X$ be a computable metric space.

(1) A finite sum of right-c.e. functions on $X$ is right-c.e.

(2) If $f: X \rightarrow \mathbb{R}$ is right-c.e. and $q \geqslant 0$ is a rational number, then $q f$ is right-c.e.

(3) If $T: X \rightarrow X$ is computable and $f: X \rightarrow \mathbb{R}$ is right-c.e., then $f \circ T$ is right-c.e. 
Furthermore, all these closure operations are uniform: a right-c.e. index for $f+g$ can be effectively obtained from right-c.e. indices for $f$ and $g$; for $f \circ T$, from a right-c.e. index for $f$ and a computable index for $T$; etc.

Proof. For $(1),(f+g)(x)<q$ if and only if there are rational numbers $s$ and $r$ such that $f(x)<s, g(x)<r$ and $r+s \leqslant q$. (2) is immediate. For $(3),(f \circ T)^{-1}(-\infty, q)=$ $T^{-1}\left[f^{-1}(-\infty, q)\right]$, and since $T$ is computable, $T^{-1}$ preserves c.e. open sets.

Corollary 12. Let $X$ be a computable metric space, let $T: X \rightarrow X$ be computable, and let $f: X \rightarrow \mathbb{R}$ be right-c.e. Then the sequence $\left\langle f^{(n)}\right\rangle$ is uniformly right-c.e.

We are ready to prove Proposition 8 , Let $(X, \mu)$ be a computable probability space. Let $P$ be an effectively closed subset of $X$, and let $x \in X$ be a Poincaré point for $T$ with respect to the collection of effectively closed subsets of $X$. We need to show that $\liminf _{n} 1_{P}^{(n)}(x) \geqslant \mu(P)$.

In our proof we make use of another classical result (see [5]):

Maximal Ergodic Theorem. Let $(X, \mu)$ be a probability space, let $T: X \rightarrow X$ be measure preserving, and let $f \in L^{1}(X)$. Let

$$
E=\left\{y \in X: f^{(n)}(y) \geqslant 0 \text { for some } n<\omega\right\} .
$$

Then

$$
\int_{E} f d \mu \geqslant 0
$$

If $\mu(P)=0$, we are done, so we assume that $\mu(P)>0$. Let $q<\mu(P)$ be a positive rational number; we show that $\liminf _{n} 1_{P}^{(n)}(x) \geqslant q$. Define $g: X \rightarrow \mathbb{R}$ by letting $g(y)=q-1_{P}(y)$. Note that for all $n<\omega$ and $y \in X, g^{(n)}(y)=q-1_{P}^{(n)}(y)$. Let

$$
E=\left\{y \in X: 1_{P}^{(n)}(y) \leqslant q \text { for some } n<\omega\right\} .
$$

By the maximal ergodic theorem,

$$
\int_{E} g d \mu \geqslant 0
$$

Lemma 13. $\mu(E)<1$.

Proof. Suppose, for a contradiction, that $\mu(E)=1$. Then

$$
\int_{E} 1_{P} d \mu=\mu(P)
$$

and

$$
\int_{E} q d \mu=q
$$

Then

$$
0 \leqslant \int_{E} g d \mu=q-\mu(P)<0
$$

by the choice of $q$, giving us a contradiction.

In fact, calculations show that $\mu(E) \leqslant(1-\mu(P)) /(1-q)$. Now $E$ may not be c.e. open, but a close associate of $E$ is. Let

$$
F=\left\{y \in X: 1_{P}^{(n)}(y)<q \text { for some } n<\omega\right\} .
$$


Lemma 14. $F$ is a c.e. open subset of $X$.

Proof. Since $P$ is effectively closed, by Example $10,1_{P}$ is a right-c.e. function. By Corollary 12, the sequence $\left\langle 1_{P}^{(n)}\right\rangle$ is uniformly right-c.e.; the result follows.

It follows that $X-F$ is an effectively closed subset of $X$. Since $F \subseteq E$, by Lemma 13 $\mu(F)<1$, so $X-F$ has positive measure. Since $x$ is a Poincaré point for $T$ with respect to all effectively closed subsets of $X$, there is an $n<\omega$ such that $T^{n}(x) \notin F$. That is, $1_{P}^{(m)}\left(T^{n}(x)\right) \geqslant q$ for all $m<\omega$. Now for all $m<\omega$,

$$
1_{P}^{(n+m)}(x) \geqslant 1_{P}^{(m)}\left(T^{n}(x)\right) \frac{m}{m+n}
$$

and so for all $m<\omega$,

$$
1_{P}^{(n+m)}(x) \geqslant q \frac{m}{m+n} .
$$

As $m /(m+n) \rightarrow 1$, we see that $\liminf _{m} 1_{P}^{(m)}(x) \geqslant q$ as required. This concludes the proof of Proposition 8

Now we prove Theorem[7. We use the fact that the measure $\mu$ is effectively outer regular.

Lemma 15. Let $(X, \mu)$ be a computable probability space. Then for all $\varepsilon>0$, for any effectively closed $P \subseteq X$ there is a c.e. open $A \supseteq P$ such that $\mu(A-P)<\varepsilon$.

Proof. This follows from the fact that the measure $\mu$ on $X$ is $\sigma$-additive and the fact that the topology on $X$ originates from a metric.

Let $\left\langle s_{i}\right\rangle$ be the sequence of "ideal" (or "rational") points of $X$. If $B=B\left(s_{i}, q\right)$ is a basic open ball, then the corresponding closed ball

$$
\bar{B}=\bar{B}\left(s_{i}, q\right)=\left\{z \in X: d\left(z, s_{i}\right) \leqslant q\right\}
$$

is, uniformly in $i$ and $q$, effectively closed, as its complement is the union of all basic open balls $B\left(s_{j}, r\right)$ where $r<d\left(s_{i}, s_{j}\right)-q$; the collection of such $s_{j}$ and rational $r$ is c.e. because $r$ and $q$ are rational numbers and $d\left(s_{i}, s_{j}\right)$ is computable.

Let $P \subseteq X$ be effectively closed; there is a c.e. set $S \subseteq \omega^{2}$ such that

$$
X-P=\bigcup_{(j, q) \in S} B\left(s_{j}, q\right) .
$$

Let $\left\langle S_{s}\right\rangle$ be an effective enumeration of $S$. We let

$$
F_{s}=\bigcup_{(j, q) \in S_{s}} \bar{B}\left(s_{j}, q-2^{-s}\right)
$$

By [6], the intersection of finitely many c.e. open subsets of $X$ is a c.e. open set, so the union of finitely many effectively closed sets is effectively closed. It follows that every $F_{s}$ is effectively closed. We have $X-P=\bigcup_{s} F_{s}$. Since $\mu$ is $\sigma$-additive, for all $\varepsilon>0$ there is an $s$ such that $\mu\left(F_{s}\right)>\mu(X-P)-\varepsilon$. Then $X-F_{s}$ is c.e. open, contains $P$, and $\mu\left(X-F_{s}\right)-\mu(P)<\varepsilon$.

For the proof of Theorem 7 , let $(X, \mu)$ be a computable probability space, let $T: X \rightarrow X$ be computable and measure preserving, and let $P \subseteq X$ be effectively closed. Let $x \in X$ be a Poincaré point for $T$ with respect to effectively closed sets. We want to show that $\lim _{n} 1_{P}^{(n)}(x)=\mu(P)$. By Proposition $8, \liminf _{n} 1_{P}^{(n)}(x) \geqslant$ $\mu(P)$, so it only remains to be shown that $\lim _{\sup } 1_{P}^{(n)}(x) \leqslant \mu(P)$. 
Let $\varepsilon>0$; by Lemma 15, let $A \supseteq P$ be c.e. open such that $\mu(A-P)<\varepsilon$. By Proposition 8

$$
\liminf _{n} 1_{X-A}^{(n)}(x) \geqslant \mu(X-A)=1-\mu(A) .
$$

Since for all $n, 1_{A}^{(n)}(x)+1_{X-A}^{(n)}(x)=1$, we get that

$$
\limsup _{n} 1_{A}^{(n)}(x)=1-\liminf _{n} 1_{X-A}^{(n)}(x) \leqslant \mu(A) .
$$

Since $P \subseteq A$, for all $n, 1_{P}^{(n)}(x) \leqslant 1_{A}^{(n)}(x)$. It follows that

$$
\limsup _{n} 1_{P}^{(n)}(x) \leqslant \limsup _{n} 1_{A}^{(n)}(x) \leqslant \mu(A) \leqslant \mu(P)+\varepsilon .
$$

Since this inequality holds for all $\varepsilon>0$, we are done. This completes the proof of Theorem 7

\section{REFERENCES}

[1] Laurent Bienvenu, Adam Day, Mathieu Hoyrup, Ilya Mezhirov, and Alexander Shen. A constructive version of Birkhoff's ergodic theorem for Martin-Löf random points. To appear in Information and Computation.

[2] Laurent Bienvenu, Adam Day, Ilya Mezhirov, and Alexander Shen. Ergodic-type characterizations of algorithmic randomness. In 6th Conference on Computability in Europe, LNCS 6158, pp. 49-58, 2010.

[3] Laurent Bienvenu, Mathieu Hoyrup, and Alexander Shen. Personal communication.

[4] Péter Gács, Mathieu Hoyrup, and Cristóbal Rojas. Randomness on computable probability spaces - a dynamical point of view. Theory Comput. Syst. 48(3): 465-485, 2011. MR2770804

[5] Paul R. Halmos. Lectures on ergodic theory. Chelsea Publishing Co., New York, 1960. MR0111817 (22:2677)

[6] Mathieu Hoyrup and Cristóbal Rojas. Computability of probability measures and Martin-Löf randomness over metric spaces. Inform. and Comput., 207(7):830-847, 2009. MR2519075 (2011b:03066)

[7] Antonín Kučera. Measure, $\Pi_{1}^{0}$-classes and complete extensions of PA. In Recursion theory week (Oberwolfach, 1984), volume 1141 of Lecture Notes in Math., pages 245-259. Springer, Berlin, 1985. MR.0820784 (87e:03102)

[8] Cristóbal Rojas. Algorithmic randomness and ergodic theory, May 2010. 5th Conference on Logic, Computability and Randomness.

Department of Mathematics, 6188 Kemeny Hall, Dartmouth College, Hanover, New HAMPSHIRE 03755-3551

E-mail address: johannaf@gauss.dartmouth.edu

School of Mathematics, Statistics, and Operations Research, Victoria University of Wellington, P.O. Box 600, Wellington, 6140 New Zealand

E-mail address: greenberg@mcs.vuw.ac.nz

Department of Mathematics, University of Wisconsin, 480 Lincoln Drive, Madison, WiSCONSIN 53706-1388

E-mail address: jmiller@math.wisc.edu

Department of Mathematics, University of Wisconsin, 480 Lincoln Drive, Madison, WISCONSIN 53706-1388

E-mail address: selwynng@math.wisc.edu 